\title{
ЗНАЧИМОСТЬ МОЛЕКУЛЯРНЫХ ПУТЕЙ АКТ/МТОR И WNT/В-САТЕNIN В КАНЦЕРОГЕНЕЗЕ ФОЛЛИКУЛЯРНОГО ЭПИТЕЛИЯ ЩИТОВИДНОЙ ЖЕЛЕЗЫ
}

\author{
Саприна Т.В., Михайлова А.А., Шестаков А.В.
}

ФГБОУВО «Сибирский государственный медицинский университет» Минздрава России, Томск

\begin{abstract}
АКТУАЛЬнОСТь: перспективным направлением для дооперационной дифференциальной диагностики рака щитовидной железы (РЩЖ) представляется изучение новых молекулярных маркеров.

К настоящему моменту недостаточно изучено влияние внутриклеточных сигнальных систем AKT/mTOR и WNT/b-catenin на онкогенез в ткани щитовидной железы, а также возможные эпигенетические механизмы регулирования активности данных путей, в частности - регуляторные воздействия микроРНК.

ЦЕЛЬ: расширение знаний о фундаментальной значимости молекулярных путей AKT/mTOR и WNT/b-catenin в канцерогенезе щитовидной железы, а также выявление связи между нарушениями эпигенетического контроля микроРНК и дисфункцией названных молекулярных механизмов.

\section{МАТЕРИАЛЫ И МЕТОДЫ.}

1. Поиск основных белков-участников сигнальных путей AKT/mTOR и WNT/b-catenin, кодирующих их генов и мРНК; создание электронной базы «белок-ген-мРНК» для названных путей;

2. Биоинформационный поиск микроРНК с помощью открытых инструментов (Targetscan, DIANAtools, miRanda) по отобранным ранее белковым мишеням. Проверка найденных микроРНК на степень эмпирической изученности по системам PubMed и DIANAtools;
\end{abstract}

3. Забор образцов ткани из послеоперационного материала пациентов с диагностированным РЩЖ с узлом в одной доле (в соответствии с критериями включения и исключения). В качестве материала исследования выступит ткань узла; контроль - отдаленная ткань не измененной доли щитовидной железы того же пациента;

4. Оценка активности сигнальных путей, а также уровня экспрессии отобранных микроРНК в образцах ткани РЩЖ и в контрольных образцах методом ПЦР в реальном времени.

5. Комплексный статистический анализ.

PЕЗУЛЬТАТЫ: произведена оценка уровня экспрессии генов, функционально активных в отношении сигнальных путей AKT/mTOR и WNT/b-catenin и регулируемых отобранными микроРНК. По результатам произведенного биоинформационного поиска предполагается оценка следующих генов: FZD6, FZD8, FZD10, WNT2, WNT7A, WNT16, DVL3, PTEN, PIK3CD, AKT1, AKT2, TSC1, RPS6KB1. В настоящее время (данные на февраль 2020 г.) производится оценка уровней активности названных сигнальных путей. По результатам произведенного биоинформационного поиска предполагается оценка экспрессии следующих мРНК: NM_001164615.2; NM_031866.2; NM_007197.3; NM_003391.3; NM_004625.4; NM_016087.2; NM_004423.4; NM_000314.7; NM_001350234.2; NM_001014432.1; NM_001243028.3; NM_000368.4; NM_001272044.1;

а также следующих микроРHK: hsa-miR-199a-5p; hsa-miR-6838-5p; hsa-miR-204-5p; hsa-miR-103a-3p; hsamiR-25-3p; hsa-miR-199b-5p; hsa-miR-124-3p.2; hsa-miR-325-3p; hsa-miR-137; hsa-miR-424-5p; hsa-miR-200a3p; hsa-miR-181d-5p.

Выводы: по итогу будут сформированы выводы относительно вовлеченности молекулярных путей AKT/mTOR и WNT/b-catenin и соответствующих микроРНК в процессы онкогенеза щЖ, что позволит расширить имеющиеся фундаментальные знания о канцерогенезе. В частности, рассмотрение различных гистотипов РЩЖ позволяет предположить возможное разделение уже существующих вариантов заболевания на дополнительные группы по степени активности изучаемых сигнальных путей. Кроме того, большое клиническое значение будет иметь оценка перспективности дальнейшего изучения выбранных маркеров для молекулярного тестирования образцов тканей с неопределенным цитологическим заключением. 